\title{
JAK inhibition with tofacitinib curbs RANKL-induced joint damage
}

Although the selective Janus kinase (JAK) inhibitor tofacatinib has demonstrated efficacy in phase III trials for rheumatoid arthritis, the mechanisms by which it influences structural joint damage are poorly understood. New research in animal studies and human cells published in the journal Arthritis \& Rheumatism now sheds light on the actions of this drug, and suggests a link between JAK signalling, production of receptor activator of nuclear factor $\mathrm{\kappa B}$ ligand (RANKL; also known as TNF ligand superfamily member 11) by $\mathrm{T}$ cells and osteoclast-mediated bone resorption.

In a rat model of adjuvant-induced arthritis, oral administration of tofacitinib once daily for 7 days greatly reduced hind-paw oedema, inflammation and osteoclast-mediated bone resorption in rats with established, destructive polyarthritis. These improvements in disease activity correlated with reduced levels of inflammatory mediators including IL-6, which decreased in both

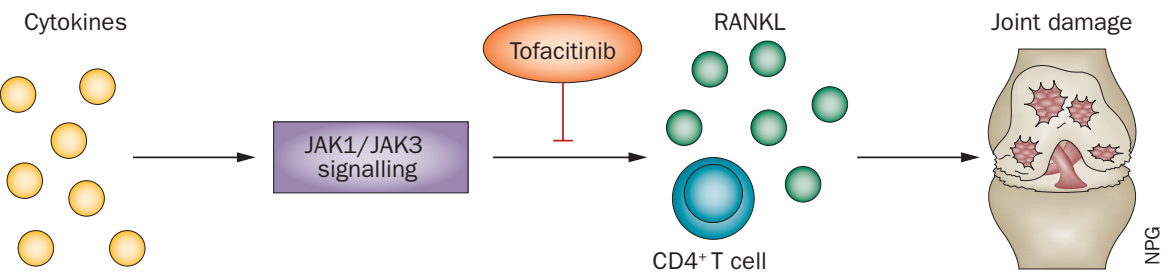

plasma and tissue within $4 \mathrm{~h}$ of treatment. Levels of RANKL in paw tissue were also decreased, but over a longer time period (after 4-7 days of treatment with tofacitinib). In parallel with these changes, substantial decreases were observed in numbers of cells expressing CD68, a marker of histiocytic cells, and $\mathrm{CD} 3$, a marker of $\mathrm{T}$ cells, 7 days after the start of treatment.

In vitro experiments in human osteoclasts suggested that inhibition of JAK1 and JAK 3 by tofacitinib is unlikely to prevent bone resorption through a direct effect on osteoclastogenesis, as treatment with tofacitinib did not influence the differentiation or function of these cells.
Notably, however, tofacitinib potently inhibited the production of RANKL by activated human $\mathrm{CD} 4^{+} \mathrm{T}$ cells in vitro, in a concentration-dependent manner.

Together, the results of the study suggest that tofacitinib suppresses osteoclast-mediated structural damage to the arthritic joint by decreasing production of RANKL by T cells.

Sarah Onuora

Original article LaBranche, T. P. et al. JAK inhibition with tofacitinib suppresses arthritic joint structural damage through decreased RANKL production. Arthritis Rheum. doi:10.1002/art.34649 